\title{
FORMAÇÃO DE DOUTORES NO BRASIL E NO EXTERIOR: IMPACTOS NA PROPENSÃO A MIGRAR
}

\author{
Milena Yumi Ramos* \\ LEA VELHO**
}

\begin{abstract}
RESUMO: Este texto reflete sobre a formação de doutores no Brasil e no exterior e o engajamento desses recursos humanos qualificados nos fluxos migratórios internacionais. Tal questão é observada a partir dos estudos sobre brain drain e desenvolvimentos teóricos posteriores, incluindo as concepções mais modernas de circulação de talentos e diásporas intelectuais. Verifica-se que a propensão de doutorandos brasileiros a migrar tem sido, historicamente, e ainda é comparativamente baixa. Mudanças recentes na política de formação de doutores no Brasil, reduzindo a oferta de bolsas para treinamento pleno no exterior, vêm reforçando essa tendência. Os impactos dessa política na dinâmica de produção de conhecimento no país e sua influência nas oportunidades de inserção de pesquisadores brasileiros em redes globais de pesquisa permanecem, no entanto, desconhecidos.
\end{abstract}

Palavras-chave: Política de formação de doutores. Circulação de cérebros. Brasil.

\section{DOCTORAL TRAINING IN BRAZIL AND ABROAD: IMPACTS ON THE PROPENSITY TO MIGRATE}

\begin{abstract}
This paper discusses the Brazilian policies for doctoral training and their impact on international migration flows. The issue is analysed in the light of the brain drain studies, including modern conceptions of talent circulation and intellectual Diaspora. Yet, the text points outs that the propensity of Brazilian doctoral students to migrate has always been low, in comparative terms. And the recent changes in doctor training policies, which reduce the offer of scholarships for full studies abroad, has made this situation even worse. Nevertheless, so far, the impacts of such policy on the dynamics of knowledge production in Brazil as well as on the opportunities for Brazilian scientists to integrate global research networks have remained unknown.
\end{abstract}

Key words: Doctoral studies. Human resources development policy. Brain circulation. Brazil.

\footnotetext{
* Doutoranda em Política Científica e Tecnológica e analista de Indicadores de Ciência, Tecnologia e Inovação na Fundação de Amparo à Pesquisa do Estado de São Paulo (FAPESP). E-mail: ramos.my@gmail.com

** PhD em Science and Technology Policy e professora titular do Departamento de Política Científica e Tecnológica do Instituto de Geociências, Universidade Estadual de Campinas (UNICAMP). E-mail: velho@ige.unicamp.br
} 


\title{
Formation DE DOCTEuRs AU BRÉSIL ET À L'ÉTRANGER: EFFETS SUR LA PROPENSION À MIGRER
}

\begin{abstract}
RÉSUMÉ: Ce texte se penche sur la formation de docteurs au Brésil et à l'étranger et l'engagement de ces ressources humaines qualifiées dans les flux migratoires internationaux. Cette question est examinée à partir des études sur le brain drain et leurs développements théoriques postérieurs, y compris les conceptions plus modernes de circulation de talents et de diasporas intellectuelles. L'article montre que la propension de doctorants brésiliens à migrer a toujours été historiquement basse, comparativement. Les changements récents dans la politique de formation de docteurs au Brésil, qui réduisent les offres de bourses de formation complète à l'étranger, renforcent cette tendance. Les effets de cette politique sur la dynamique de production de connaissance dans ce pays et pour les occasions d'insertion de chercheurs brésiliens dans les réseaux mondiaux de recherche continuent cependant inconnus.
\end{abstract}

Mots-clés: Politique de formation de docteurs. Circulation des compétences. Brésil.

\section{Introdução}

$\mathrm{E}$ ste artigo tem o objetivo de contribuir para a reflexão sobre a relação das políticas de formação de recursos humanos altamente qualificados, particularmente de doutores, com a circulação internacional de tais pesquisadores. Busca-se desenvolver o argumento de que o foco recente da política brasileira na formação quase que exclusiva de doutores em instituições nacionais carece de evidências que lhe deem sustentação e legitimidade. Além disso, argumenta também que tais políticas adotam uma concepção ultrapassada de que migração é, necessariamente, sinônimo de perda de cérebros, num momento em que o referencial analítico atual indica a importância da mobilidade e da circulação de cérebros para a produção de conhecimento e inserção científica internacional.

Para isso, esse artigo está organizado em cinco seções, além desta introdução. A primeira trata do contexto em que a mobilidade de pessoas qualificadas se tornou um problema de pesquisa e de interesse da política. Na seção seguinte, traça-se um breve panorama da evolução do entendimento sobre esta mobilidade, desde os estudos que cunharam o termo brain drain até a compreensão atual do fenômeno como circulação internacional de talentos. As seções 3 e 4 fornecem dados dos países desenvolvidos e do Brasil sobre a mobilidade de talentos científicos, numa tentativa de mostrar como esses países lidam com a questão. A seção final contém uma síntese das ideias apresentadas e aponta algumas implicações para o Brasil. 


\section{Demanda e oferta de recursos humanos qualificados: onde se encontram?}

Nas últimas duas décadas, tem se intensificado o debate político e acadêmico acerca da migração, sobretudo da parcela da população considerada de alta qualificação, entendida como aquela formada por pessoas de talento e criatividade em ciência e tecnologia, negócios, artes e cultura e outras atividades, conforme Solimano (2006a). Fatores como a transição demográfica que afeta os países avançados e o aumento dos estímulos à mobilidade internacional estão no centro desse debate.

Vista sob a perspectiva da política científica e tecnológica, a questão volta-se para o impacto do envelhecimento da população sobre a força de trabalho com alta qualificação, a pouca atratividade das profissões científicas para os jovens de hoje e os efeitos de ambos os fatores sobre os fluxos migratórios globais. Evidentemente, os instrumentos de política acionados para enfrentar o problema variam, grosso modo, entre os países desenvolvidos e aqueles em desenvolvimento, na medida em que estão em lados opostos do problema.

Nos países desenvolvidos, o ritmo de entrada de novos indivíduos qualificados tem sido menos acelerado do que o envelhecimento da população já existente, resultando na elevação da idade média desse contingente populacional estratégico. Na União Europeia, por exemplo, a parcela dos recursos humanos em ciência e tecnologia na faixa etária de 45 a 64 anos é de 35\%, superior aos $31 \%$ da coorte 25 a 34 anos. A taxa de crescimento dos trabalhadores que desenvolvem atividades científicas nessas duas faixas etárias entre 2001 e 2007 reforça a tendência de elevação da idade média: enquanto na coorte 25 a 34 anos foi registrado um crescimento médio de $2,77 \%$ ao ano, na faixa etária entre 45 e 64 anos, este foi de $4,02 \%$ ao ano (eurostat, 2009).

A persistência desse padrão é vista como uma ameaça à capacidade interna de prover talentos e habilidades necessárias para sustentar, no longo prazo, o crescimento da economia de países desenvolvidos (Mahroum, 2000). Projeções indicam uma redução da oferta de mão de obra de cerca de seis milhões de pessoas em 2020 (CEDEFor, 2008). Portanto, as novas gerações que entrarão no mercado de trabalho nos próximos anos provavelmente não suprirão a demanda por trabalhadores qualificados. Esse cenário tem implicações diretas para as políticas de educação e treinamento, de atração de talentos estrangeiros e de mobilidade regional/internacional.

O problema torna-se ainda mais grave porque já foi detectado um interesse decrescente dos jovens em seguirem carreiras científicas e das engenharias (NAS, 
1995) devido a vários fatores. Por um lado, muitos jovens consideram o ensino de ciências pouco interessante e muito difícil (EURAB, 2002). Eles também percebem um aumento da crítica, por parte do governo e da sociedade, em relação à qualidade e adequação do ensino superior e avançado e à baixa relevância (econômica e social) do conhecimento produzido pelos pesquisadores (Balbachevsky, [2001]).

Por outro lado, a atratividade das carreiras depende do grau de estabilidade e continuidade que elas proporcionam (NAS, 1995). Com frequência, as carreiras científicas são percebidas pelos jovens como demasiadamente especializadas para possibilitar oportunidades futuras mais amplas, e o seu desenvolvimento é excessivamente exigente e não compensado por garantia de emprego ou por salários mais altos.

Em fases mais avançadas das carreiras científicas também existem gargalos. Jovens cientistas frequentemente são recrutados, com base em contratos temporários de curto prazo, para apoiar a execução de projetos de pesquisa específicos (EC, 2008). Isso restringe as chances de jovens talentos fazerem a transição e se tornarem pesquisadores independentes. Somem-se a isso os esquemas atípicos de remuneração aos quais eles frequentemente estão submetidos: bolsas e auxílios com acesso limitado aos benefícios da seguridade social. Como resultado, recém-formados em áreas científicas e tecnológicas estão sendo crescentemente atraídos por outras carreiras, como aquelas ligadas ao sistema financeiro, à administração de negócios e ao setor imobiliário, que oferecem melhores salários e condições de trabalho e, talvez, status profissional e social superior ao de carreiras em ciência e tecnologia (NAS, 1995).

Essa situação já tem reflexos na composição das vagas preenchidas no ensino superior em países desenvolvidos, tanto em nível de graduação como de pós-graduação: a proporção de estudantes nacionais relativamente à de estrangeiros tem diminuído. Muitos estudantes de países em rápido desenvolvimento, principalmente chineses, coreanos e indianos - que reconhecidamente têm motivações mais acentuadas para carreiras de ciência e tecnologia -, acabam ocupando vagas em cursos de graduação e pós-graduação em ciências e engenharia oferecidos por universidades nos Estados Unidos, Canadá, Austrália, Reino Unido, Alemanha e França.

Assim, em vários países desenvolvidos o fluxo de jovens do ensino médio para o ensino superior não tem aumentado nos anos recentes, ao passo que naqueles em desenvolvimento os estudantes têm encontrado fortes estímulos à qualificação. Na China, o número de alunos que completaram a graduação dobrou entre 1995 e 2005 e o de titulados na pós-graduação cresceu $24 \%$ ao ano entre 2000 e 2005 (OECD, 2008). Na Índia, em princípios dos anos de 1990, havia 5,3 milhões de estudantes matriculados no ensino superior; este número expandiu para 7,7 milhões no final da década. Já o número de titulados no doutorado subiu de 8.383 em 1990-91 para 10.951 em 1998-99 (Khadria, 2004). No Brasil, o número de concluintes no ensino 
superior aumentou de 300.761 em 1998 para 800.318 em 2008 (Brasil, 2009); o de titulados no mestrado cresceu de 12.351 em 1998 para 33.360 em 2008; e o de titulados no doutorado, de 3.915 para 10.711 nesse período (CAPES, 2009).

As bem-sucedidas políticas públicas de formação de pessoal de nível superior nesses países, no entanto, com variações relevantes de um país para outro, não têm sido complementadas por políticas de promoção da absorção desse contingente pelo mercado de trabalho local em ocupações de qualificação compatível, capazes de propiciar as oportunidades profissionais e pessoais almejadas por esses indivíduos. Tampouco outros agentes do sistema nacional de inovação desses países, sobretudo as empresas, realizam esforços inovadores que justifiquem a incorporação de parcela significativa da população nacional altamente qualificada. No Brasil, por exemplo, menos de três mil pós-graduados estavam ocupados em atividades de pesquisa e desenvolvimento nas empresas em 2000, ano no qual o país titulou mais de 18 mil mestres e cinco mil doutores. Em 2005, somente 1.189 doutores ocupavam posições em atividades internas de P\&D1 nas empresas (IBGE, 2007).

Combinados, a demanda por mão de obra qualificada nos países desenvolvidos e o crescimento da oferta de pessoal de nível superior nos países em desenvolvimento têm concorrido para a concepção, por parte do primeiro grupo, de políticas de atração e retenção de talentos, particularmente de doutores, provenientes dos países em desenvolvimento.

Enquanto nos países desenvolvidos o aproveitamento desses fluxos tem sido objeto de pesquisa e de políticas ativas por parte dos governos nacionais há vários anos, nos países em desenvolvimento ainda há pouca evidência sobre a dimensão, as motivações e os efeitos da mobilidade internacional de pessoal altamente qualificado. A tendência dos países em desenvolvimento tem sido criar medidas para coibir a imigração de seus talentos, tais como penalidades financeiras, e evitar contato prolongado dos jovens pesquisadores locais com grupos de países desenvolvidos. Isso, a despeito da falta de conhecimento da extensão e do impacto, no médio e longo prazo, da migração e das medidas para evitá-la. Afinal, que parcela do contingente de pessoal qualificado migra? Quais os condicionantes desta decisão? Seria, de fato, migração ou mera circulação? Que impactos as políticas para evitar a migração têm na dinâmica de produção de conhecimento dos países em desenvolvimento?

A carência de evidências empíricas e analíticas que informem regularmente a formulação e avaliação de políticas públicas nesses países pode impedir que eles se beneficiem plenamente do potencial existente em termos de cooperação científica, transferência de tecnologia, formação de alto nível, aquisição de conhecimentos codificados e tácitos e a inserção internacional dos futuros pesquisadores, entre outros. Além disso, deve-se considerar que existem várias maneiras de entender a mobilidade 
de recursos humanos e cada uma delas informa políticas e programas diferenciados. Alguns destes modos de entender são analisados na próxima seção.

\section{Do brain drain à circulação internacional de talentos}

Por volta de meados de 1960, com o pós-guerra e a Guerra Fria, as atividades científicas e tecnológicas começaram a responder à relação centro-periferia típica do imperialismo capitalista. Essa relação expressou-se também nos padrões de migração internacional, sobretudo entre a população considerada qualificada: contingentes não desprezíveis de pessoas tituladas no ensino superior e pós-graduação se deslocavam do hemisfério Sul para o Norte, das regiões subdesenvolvidas para as desenvolvidas (Guimarães, 2002; Meyer, Kaplan \& Charum, 2001). Esse movimento bipolar foi entendido como perda ou êxodo permanente de cérebros, com impactos negativos para o desenvolvimento científico, tecnológico e socioeconômico do país de origem, uma vez que a capacidade de acumulação de capital, já diferenciada dos países avançados em relação aos menos desenvolvidos, perpetuava-se com o brain drain.

Esses argumentos ganharam importância internacional, levando à intensificação dos estudos sobre o tema e à criação de sistemas de acompanhamento dos deslocamentos de recursos humanos qualificados no mundo. Os governos, tanto dos países de origem quanto dos países receptores desses contingentes, passaram a se debruçar sobre as seguintes questões (Meyer, 2003):

- Qual a dimensão e a direção do fluxo dessas pessoas?

- Quais os prejuízos decorrentes da migração, que alcance têm e como podem ser evitados ou minimizados?

- Como atrair e reter essas pessoas?

- Qual é a intensidade desses deslocamentos, segundo as disciplinas científicas e profissões qualificadas?

Como resultado das investigações então levadas a cabo em alguns países, surgiu, na década de 1980, uma tese que vislumbrava os efeitos positivos do brain drain, e não apenas os negativos. Segundo os defensores dessa visão, a migração internacional de pessoas capacitadas teria efeitos positivos para o desenvolvimento global advindos da transferência de conhecimentos e capital intelectual e cultural incorporados em estudantes, cientistas e artistas (Solimano, 2006a).

A partir dessa tese adveio, em princípios dos anos de 1990, a abordagem do brain gain, que aponta os benefícios, para os países de origem, da emigração e posterior repatriação de indivíduos qualificados. Estes proporcionariam diferentes tipos 
de contribuição para o desenvolvimento do país natal, tais como o fortalecimento dos sistemas educacionais; o estímulo para inserção de talentos em mercados e redes internacionais de produção intelectual, cultural e econômica; o aproveitamento dos seus contatos e canais de acesso a recursos disponíveis no exterior (conhecimentos técnicos e tácitos, habilidades laborais, capacidade empreendedora, redes sócio-profissionais, investimentos) para o desenvolvimento dos países de origem.

Em países como Índia e China, esses resultados já são percebidos: segundo Solimano (2006b), muitos indianos e chineses que se graduaram nos Estados Unidos tornaram-se empreendedores de sucesso neste país (por exemplo, no Vale do Silício). Conseguiram, com isso, estabelecer conexões entre os mercados asiático e norte-americano e promover o contato e o acesso mútuo à tecnologia e capital em ambos os mercados. Nos anos de 1990 e início dos 2000, esses empreendedores também inauguraram novos negócios produtivos em seus países de origem, transferindo tecnologia e conhecimento de mercado.

Esses são casos de sucesso de um processo que se tornou uma tendência global: a mobilidade internacional de recursos humanos qualificados é irrefreável e está ligada às novas oportunidades e riscos oferecidos pela globalização. Não por acaso, os países desenvolvidos, inclusive alguns emergentes, com destaque para a China e a Índia, incluíram entre suas prioridades estratégicas neste início de século sua atratividade para estudantes de alto nível e pesquisadores estrangeiros.

Tal mobilidade tem efeitos distintos em diferentes países, dependendo de vários fatores. Precisamente por isso, a tensão entre a capacitação nacional das gerações futuras de profissionais e cientistas e a necessidade de treiná-los, de modo a permitir sua inserção internacional, se estabelece e impõe um desafio importante aos tomadores de decisão (Nerad, 2006).

No centro dessa política, nas economias baseadas no conhecimento, estão os talentos científicos. Os fluxos migratórios desse contingente se orientam aos centros com maior intensidade de conhecimento - que fixam os padrões e paradigmas. Atualmente, no entanto, os centros de atração não correspondem exatamente às relações centro-periferia do pós-guerra, mas são numerosos e estão dispersos pelos países do Norte e do Sul. Tampouco os fluxos migratórios se dão simplesmente entre um país de origem e um de destino; agora, as possibilidades de deslocamentos geográficos internacionais são múltiplas e configuram movimentos de circulação que obedecem à hierarquia internacional das relações científico-tecnológicas (Balán, 2008; Davenport, 2004; Meyer, Kaplan \& Charum, 2001).

Diante desse novo cenário, diversos países revisaram suas políticas de formação de recursos humanos e passaram a adotar medidas de estímulo controlado à mobilidade internacional de talentos técnicos e científicos, incluindo a formação e 
exploração de redes de diáspora intelectual. Estas últimas vêm a ser redes de expatriados muito diversas, mas com finalidades e objetivos muito parecidos: fomentar ações coordenadas entre os membros para promover interesses coletivos e ajudá-los a enfrentar desafios comuns e a se reinserirem no país de origem, sem necessariamente regressarem (Meyer \& Brown, 1999).

Os expatriados qualificados passaram a ser percebidos não como uma perda permanente, mas sim como recurso potencial para o país, seja no regresso definitivo ou na circulação internacional, que inclui seu país de origem. A expectativa é de que as transferências tangíveis e intangíveis que eles são capazes de realizar contribuam para o desenvolvimento de seu país de origem.

A intensidade desses efeitos está associada ao tamanho e nível de qualificação da comunidade nacional e de expatriados. Como destaca Moura Castro (2007), o ideal é alcançar um equilíbrio adequado entre o tamanho da comunidade de expatriados e o tamanho da capacidade local, de forma que os países de origem possam se beneficiar da ausência temporária de parte dos cidadãos qualificados, em cuja formação a nação investiu tempo e montantes consideráveis.

\section{Mobilidade internacional de talentos científicos nos países da OCDE}

Nas duas últimas décadas, a imigração de pessoal qualificado para os países desenvolvidos vem aumentando nitidamente como resposta a políticas claras de atração, nas quais os pesquisadores e estudantes de pós-graduação ocupam uma posição de destaque:

\footnotetext{
Virtualmente todos os países membros da ocDe [Organização para a Cooperação e Desenvolvimento Econômico] preveem que haverá necessidade de imigrantes altamente qualificados nos próximos anos, especialmente em carreiras científicas e tecnológicas (...). A mobilidade internacional de estudantes, em particular, parece um modo promissor de expandir a imigração de pessoal altamente qualificado, sobretudo porque coortes mais jovens estão em declínio em muitos países da OCDE. (OECD, 2009, p. 161; nossa tradução)
}

Os instrumentos de atração desses talentos científicos incluem a concessão de vistos especiais e políticas de absorção pelo mercado de trabalho. No entanto, o instrumento preferencial tem sido a "porta acadêmica", por meio da oferta de formação de pós-graduação e atividades temporárias de docência e pesquisa. Entende-se que estas proporcionam o tempo necessário para socializar o indivíduo no ambiente local, treiná-lo para exercer atividades com elevada exigência de qualificação e avaliálo quanto à disposição e condições de permanência. 
Como resultado, o número de estudantes de pós-graduação titulados em universidades dos Estados Unidos - o país que mais recebe estudantes estrangeiros cresceu quase $340 \%$ de 1978 a 2008, em todas as áreas do conhecimento (NSF, 2009a). Nas engenharias e ciências físicas, esse crescimento foi ainda maior ( $474 \%$ e $463 \%$, respectivamente), o que parece indicar que as carreiras científicas têm perdido atratividade para os jovens norte-americanos, criando oportunidades para os talentos dos países em desenvolvimento.

Esse treinamento pode resultar em desdobramentos para os estudantes estrangeiros, levando, por exemplo, ao pós-doutoramento. De fato, o número de estudantes estrangeiros titulados nos Estados Unidos que prosseguiram seus estudos em nível de pós-doutorado aumentou 20\%, entre 2000 e 2007 (NSF, 2009b). Esses dados corroboram a tendência de diminuição do interesse de jovens locais em seguirem carreiras científicas, abrindo espaço para estudantes estrangeiros dispostos a permanecer por mais tempo no país que os recebeu, a despeito dos rendimentos menores e das condições mais precárias de trabalho e de seguridade social a que ficam submetidos.

Menos ativa que os Estados Unidos na atração de imigrantes altamente qualificados, ainda assim a Europa (UE15) atrai cerca de um quarto do total de imigrantes qualificados circulando no mundo (Katseli, Lucas \& Xenogiani, 2006). Mais da metade dos estrangeiros vivendo nos países da UE15 provém dessa mesma região e grande parte da outra metade (26,4\%) provém de outros países europeus e da África do Norte. Portanto, ainda que se pense que a Europa é um local importante de destino para os latino-americanos qualificados, esse não é o caso.

Os números apresentados sobre a mobilidade internacional de talentos científicos podem dar a impressão - equivocada - de que as políticas de atração adotadas pelos países desenvolvidos são eficazes. Na verdade, existem dificuldades e desafios importantes que se colocam aos países receptores: muitas vezes, as qualificações e experiência profissional dos imigrantes, incluindo a competência linguística, não são devidamente reconhecidas pelos centros que os recebem, frustrando as expectativas dos imigrantes. Por isso, a admissão de estudantes estrangeiros é vista pelos países desenvolvidos como uma maneira de selecionar, com menos riscos potenciais, profissionais para suprir as necessidades locais de mão de obra altamente qualificada.

\section{Formação e circulação internacional de doutores brasileiros}

O Brasil realizou investimentos continuados e consideráveis na formação de recursos humanos em ciência e tecnologia nos últimos quarenta anos. Sustentado 
nos Planos Nacionais de Pós-Graduação (PNPG), o processo de expansão da pós-graduação resultou de planejamento e financiamento estatal, tendo as universidades públicas como base institucional. Como resultado, os cerca de 800 cursos de mestrado e doutorado da década de 1970 cresceram para quase 3.700 em 2008. Esses programas formaram, em 2008 apenas, 33.360 mestres e 10.711 doutores, com um contingente de estudantes em torno de 140 mil (CAPES, 2009).

Essa expansão quantitativa ocorreu em todas as áreas do conhecimento, porém, de forma desigual no território nacional. Em 1996, o estado de São Paulo oferecia $36,4 \%$ dos programas de mestrado e $55 \%$ dos de doutorado. Doze anos depois, a concentração havia diminuído e São Paulo oferecia 36\% dos doutorados. Ainda assim, a liderança deste estado é notável, principalmente quando se leva em consideração a presença de programas com notas mais altas (42,7\% dos programas de mestrado e doutorado avaliados com conceitos 5, 6 ou 7 pela CAPEs, em 2008, eram oferecidos em São Paulo). Esse quadro revela a importância dos fluxos migratórios internos de pessoal altamente qualificado, outra faceta da questão da circulação de cérebros que merece estudos mais aprofundados.

A estrutura acadêmica construída pelo país permitiu a ampliação significativa da comunidade científica nacional e um expressivo crescimento de sua produção intelectual. A construção relativamente rápida dessa base científica valeu-se, em grande medida, até meados dos anos de 1990, do treinamento de mestres e doutores no exterior, com o compromisso de regresso. Três mecanismos possibilitaram essa política: a concessão de bolsas com a garantia do emprego na instituição de vínculo no país; a inclusão de cláusulas determinando o retorno imediato após a obtenção do título no termo de compromisso assinado pelos bolsistas; e, mais recentemente, esforços governamentais para o estabelecimento de acordos internacionais com "países receptores" para impedir a concessão de visto de permanência a ex-bolsistas (Schwartzman, 1978; Balbachevsky \& Marques, 2009).

Schwartzman (1978) destaca também outros fatores que contribuíam para o regresso: as dificuldades com a língua estrangeira, os problemas de adaptação ao sistema educacional no exterior e os vínculos familiares mantidos no Brasil. Com isso, o país estimulou um regresso massivo dos seus recursos humanos treinados no exterior: acima de $80 \%$ imediatamente após a titulação; e outros $14 \%$ após mais alguns meses de formação acadêmica complementar (Moura Castro, 2007; Santana Lombas, 1999).

De fato, essa política proporcionou progressos importantes em escala e qualidade no sistema brasileiro de pós-graduação e pesquisa. Por outro lado, limitou o potencial de aplicação dos conhecimentos localmente produzidos. Isso porque tal política baseou-se numa visão ortodoxa de pesquisador, dominante no Brasil e em outros países latino-americanos - fortemente correspondente ao cientista das ciências duras - da 
pesquisa básica (Vessuri, 2007). O fraco interesse do setor produtivo pela capacidade de pesquisa assim construída tem aí suas raízes. Essa tendência foi reforçada pela política de avaliação e promoção aplicada ao sistema de pesquisa brasileiro, apoiada em critérios de produtividade, qualidade e relevância típicos dos segmentos mais básicos da pesquisa, aplicados homogeneamente a todas as áreas do conhecimento.

De qualquer forma, tal estratégia serviu aos propósitos de expansão e autogestão da instituição científica no Brasil e levou alguns autores (De Meis \& Longo, 1990; Meneghini, 1991) a considerarem que as instituições nacionais já eram capazes de prover a formação acadêmica adequada para futuros mestres e doutores, e ainda fazê-lo a um custo bem menor do que a formação acadêmica plena no exterior, evitando problemas de reinserção no Brasil.

\section{Tabela 1}

Bolsas da CAPES e do CNPq para formação de pesquisadores brasileiros no exterior, segundo modalidade - Brasil: 1996-2008 (anos selecionados)

\begin{tabular}{|c|c|c|c|c|c|}
\hline Ano & $\begin{array}{c}\mathbf{N}^{\circ} \\
\text { bolsistas }\end{array}$ & $\begin{array}{c}\text { Doutorado } \\
\text { pleno no } \\
\text { exterior } \\
\mathbf{( \% )}\end{array}$ & $\begin{array}{c}\text { Doutorado } \\
\text { “sanduíche” no } \\
\text { exterior } \\
\mathbf{( \% )}\end{array}$ & $\begin{array}{c}\text { Pós-doutorado } \\
\text { no exterior } \\
\mathbf{( \% )}\end{array}$ & $\begin{array}{c}\text { Outros no } \\
\text { exterior (1) } \\
\mathbf{( \% )}\end{array}$ \\
\hline $\mathbf{1 9 9 6}$ & 1279 & 74 & CAPES & & 5 \\
$\mathbf{2 0 0 0}$ & 1518 & 50 & 12 & 9 & 21 \\
$\mathbf{2 0 0 4}$ & 1567 & 46 & 20 & 9 & 12 \\
$\mathbf{2 0 0 8}$ & 4135 & 17 & 27 & 15 & 23 \\
& & & 38 & 22 & 3 \\
$\mathbf{1 9 9 6}$ & 1655 & 68 & CNPq & & 2 \\
$\mathbf{2 0 0 0}$ & 576 & 68 & 14 & 15 & 2 \\
$\mathbf{2 0 0 4}$ & 510 & 51 & 12 & 18 & 3 \\
$\mathbf{2 0 0 8}$ & 551 & 21 & 22 & 25 & 39 \\
\hline
\end{tabular}

Fonte: Coordenação de Aperfeiçoamento de Pessoal de Nível Superior (CAPEs). Sistema GEocapes; Conselho Nacional de Desenvolvimento Científico e Tecnológico (CNPq). Estatísticas e Indicadores do Fomento. Tabela 2.2.5.

(1) Inclui bolsas no exterior nas modalidades: (a) CAPEs: graduação "sanduíche", mestrado pleno e mestrado "sanduíche"; (b) cNPq: aperfeiçoamento/estágio/especialização, estágio júnior e estágio sênior.

O que se verificou, então, a partir de meados dos anos de 1990, foi uma vertiginosa queda na concessão de bolsas de doutorado pleno no exterior (Tabela 1) - ainda mais acentuada no caso do mestrado - paralelamente a um aumento na concessão de bolsas de doutorado "sanduíche" e pós-doutorado, por um período normalmente 
não superior a um ano. Em esquemas de curta duração como estes, o mais provável é que os estudantes não consigam integrar-se à comunidade local e nem participar da vida acadêmica da instituição que o recebe; tampouco aproveitam amplamente sua experiência de estudo em outro país (Velho, 2001; Schwartzman, 2009).

Não se conhecem, até o momento, estudos ou avaliações sobre os impactos dessa política para a dinâmica de produção do conhecimento e a inserção de futuros cientistas brasileiros em redes de pesquisa internacionais. Adicionalmente, as trajetórias de pesquisadores com pós-doutorado no exterior também constituem objeto de estudo ainda inexplorado.

As informações sobre a pequena parcela de doutores brasileiros no exterior - em formação ou titulados - são insuficientes e fragmentadas em estudos ocasionais (Schwartzman, 1972; 1978; De Meis \& Longo, 1990; Meneghini, 1991; Santana Lombas, 1999; Guimarães, 2002; Velloso, 2006). Sabe-se, no entanto, que são estatisticamente muito poucos, principalmente quando comparados ao contingente de doutores de outros países, inclusive competidores emergentes, tais como China e Índia (Figura 1). É evidente que, dadas as populações muito mais numerosas destes países em relação à situação brasileira, os números de doutores não são estritamente comparáveis. Entretanto, vale notar que entre 1997 e 2007, enquanto o Brasil manteve constante o número de doutores treinados nos EUA, a China e a Índia dobraram seus contingentes em treinamento.

\section{Figura 1}

Estrangeiros que receberam título de doutor em programas de pós-graduação em Ciências e Engenharia oferecidos por instituições norte-americanas - China, Índia e Brasil: 1997 e 2007
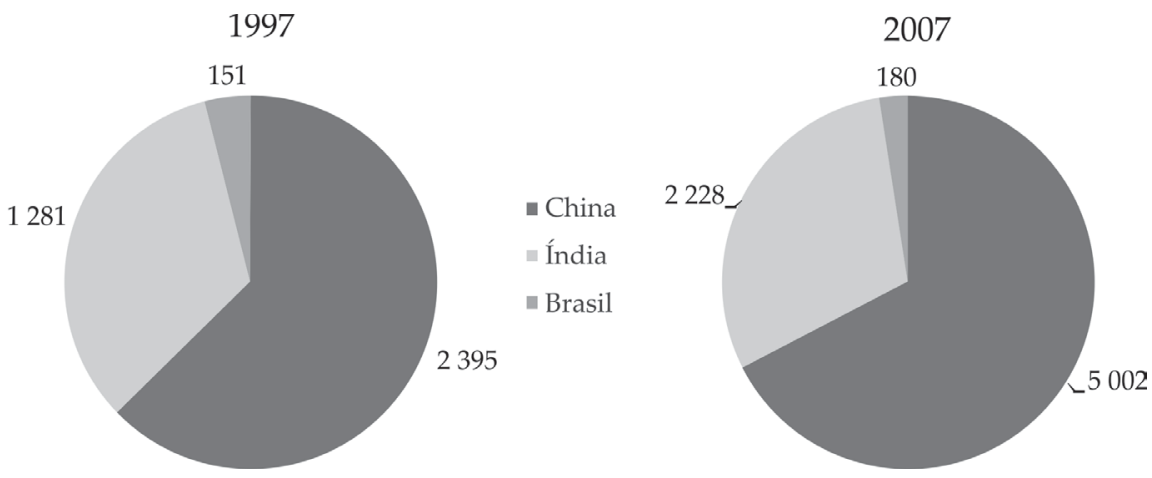

Fonte: NSF/NIH/USED/USDA/NEH/NASA (2008), Survey of Earned Doctorates. 
Muitos desses recém-doutores têm planos de ficar nos Estados Unidos: entre 2001 e $2007,90 \%$ dos chineses e $88 \%$ dos indianos titulados tinham planos de permanência. Em contraste, apenas 39\% dos brasileiros tinham planos de continuar os estudos ou trabalhar naquele país (NSF, 2009a).

Devido às punições financeiras a que estão sujeitos por descumprimento da cláusula de regresso ao país, alguns bolsistas que obtiveram sucesso na inserção no mercado de trabalho no exterior omitem sua situação, o que dificulta ainda mais o acompanhamento dos expatriados brasileiros e reduz muito sua propensão a colaborar com o país. Essa política punitiva das agências indica que, desde os anos de 1970 até hoje, a ida de pesquisadores brasileiros para trabalhar em outros países é vista necessariamente como uma perda, isto é, adota-se ainda a visão tradicional de brain drain.

Esta parece ser uma precaução infundada, já que os dados disponíveis indicam que o Brasil não é exportador líquido de talentos, em particular de doutores. Além disso, o país passou a ser destino de montantes não desprezíveis de investimentos diretos externos, por exemplo, pela implantação de unidades empresariais de grupos transnacionais, com a consequente entrada de executivos estrangeiros altamente qualificados. A dimensão exata dessa entrada, no entanto, permanece desconhecida.

Também infundado parece ser o argumento do alto custo de formação plena no exterior, já que existe a possibilidade de flexibilização do financiamento dos estudos fora do país. Schwartzman (2009) destaca a combinação de bolsas fornecidas pelo Brasil (nos dois primeiros anos, por exemplo) e a possibilidade de obtenção de financiamento no país de destino, por meio de bolsas locais ou atividades de assistência ao ensino e à pesquisa em instituições locais, em tempo parcial.

Por outro lado, o temor do brain drain não tem induzido medidas para resolver o desequilíbrio entre a formação de alto nível e a criação de oportunidades de trabalho qualificado no Brasil.

\section{Considerações finais}

Este texto apresenta uma breve descrição do brain drain, buscando retratar como o entendimento desse fenômeno foi se alterando desde o primeiro registro de uso deste termo, nos anos de 1960, até os dias de hoje. Dos estudos iniciais que apontavam sempre perdas para países de origem e benefícios para os receptores, evoluiuse para a ideia de circulação internacional de talentos. Esta mobilidade, atrelada à intensificação da globalização, é tida como um fenômeno irrefreável, do qual os países podem tirar proveito, desde que promovam a capacitação local e estabeleçam as conexões necessárias entre as comunidades nacional e de expatriados. 
O Brasil posiciona-se de forma relativamente isolada nesse contexto. Apesar da escassez de pesquisas sobre a circulação internacional de talentos brasileiros, particularmente os científicos, é possível constatar que o país tem enviado cada vez menos estudantes de doutorado ao exterior para formação plena, como mostram os dados de bolsistas apoiados pela CAPEs e pelo CNPq.

O mais preocupante, entretanto, são as práticas punitivas adotadas pelas agências de fomento à pesquisa no Brasil para os poucos doutores que decidem permanecer no exterior após a titulação. Elas revelam que o sistema de formação de doutores no país ainda não absorveu os conceitos mais modernos sobre a importância e os potenciais benefícios da circulação internacional de talentos científicos.

Essa situação pode ter consequências indesejáveis para o país no longo prazo. Indícios de isolamento e paroquialismo já podem ser percebidos. Estudo recente (Heidrick \& Struggles 2007, p. 3) mostra que, no que diz respeito à capacidade de formar talentos internacionalmente competitivos no longo prazo:

(...) a amigável história dos BRIC frequentemente repetida na mídia - sobre a inexorável ascensão de Brasil, Rússia, Índia e China - é mais precisamente expressa como uma história de sucesso dos IC. (...) Índia e China estão entre os 10 primeiros $\left(10^{\mathrm{a}}\right.$ e $6^{\mathrm{a}}$ posições em 2007 e 2012, respectivamente), enquanto a Rússia ocupa a 18a posição em 2007 e 2012, e o Brasil cai da 23 a para a 25a posição em cinco anos. (Tradução nossa)

Especificamente em relação aos doutores brasileiros, Velho (2001) e Guimarães (2002) mostram que a proporção dos que se titulam no exterior vem se reduzindo e é muito inferior à de países de industrialização tardia, tais como a Coréia, a China e a Índia.

O baixo engajamento de pesquisadores brasileiros em programas de treinamento no exterior tem reflexos também no modo como suas atividades e resultados de pesquisa são desenvolvidos e difundidos entre os pares. Glänzel, Leta e Thus (2006) revelam que a proporção das publicações em colaboração internacional com a participação de autores brasileiros permaneceu estagnada nas últimas décadas, ainda que a produção científica do país tenha crescido a taxas relativamente elevadas no período. Alguns estudiosos do assunto ressaltam inclusive que um dos maiores desafios é reverter a orientação interna da comunidade acadêmica brasileira (Balbachevsky, 2006). Segundo a autora, menos de $20 \%$ dos professores universitários brasileiros do setor público têm contato com a comunidade internacional. E, o que é mais preocupante, esse isolamento da academia brasileira não é visto como um problema pela sociedade e muito menos, aparentemente, pelos tomadores de decisão em política científica e tecnológica.

A persistência dessa tendência pode afetar as perspectivas de inserção internacional da ciência brasileira no longo prazo. Portanto, torna-se urgente a reavaliação da 
política de recursos humanos no Brasil à luz das abordagens mais modernas. O que se defende aqui é a promoção de políticas que levem em conta os benefícios do diálogo, as oportunidades de aprendizagem e de cooperação que se apresentam quando o intercâmbio e o fluxo de pessoas e ideias estão abertos, evitando o nacionalismo cultural, científico e tecnológico, com seus óbvios limites (Schwartzman, 2009).

De acordo com Velho (2001), a formação acadêmica no exterior aumenta a possibilidade de inserção de pesquisadores brasileiros em redes internacionais de produção de conhecimento, bem como de acesso privilegiado a recursos escassos no país. Além disso, a socialização do estudante ou pesquisador brasileiro no exterior permite que eles não apenas absorvam elementos codificados do conhecimento, mas, sobretudo, conhecimentos tácitos incorporados nos pares estrangeiros, tais como a dinâmica de organização de grupos de pesquisa e a maior articulação com o setor privado, ou ainda, segundo Vessuri (2007), a integração dos conhecimentos e sua aplicação no mundo real, o suporte aos tomadores de decisão e a avaliação de possíveis benefícios e obstáculos proporcionados por diferentes opções de resposta a questões centrais da atualidade.

Para ajudar a pensar sobre as opções de política possíveis para o caso brasileiro, inclusive aquelas listadas anteriormente, torna-se urgente conhecer a dinâmica da mobilidade de talentos científicos brasileiros de forma mais aprofundada, apoiada em um referencial teórico moderno. Fica aqui a sugestão de se estimularem estudos nessa direção. Como ressalta Solimano (2006b), é necessário conhecer melhor o tamanho, a direção e a composição (em termos de áreas do conhecimento e profissões) do fluxo de pessoas qualificadas, de maneira sistemática, sobretudo nos países em desenvolvimento, em que as informações são precárias ou até mesmo inexistentes. Esses países precisam aprimorar sua capacidade estatística e analítica sobre a mobilidade de talentos e, para isso, o desenvolvimento de bases de dados que apoiem ações de acompanhamento permanentes é essencial. Essa é a forma pela qual a coleta de evidências confiáveis e atualizadas, inclusive sobre a natureza da ausência - permanente ou temporária - dessas pessoas, tornar-se-ia possível, bem como avaliações mais precisas sobre os impactos de ambas as formas de mobilidade, que são bastante diferentes.

\section{Nota}

1. Referem-se ao conjunto de atividades de pesquisa e desenvolvimento executadas nas empresas de modo contínuo ou ocasional, com ou sem organização formal, independentemente da origem de seu financiamento. Entendam-se atividades de pesquisa e desenvolvimento segundo o conceito adotado pelo IBGE nas suas pesquisas de inovação tecnológica, ou seja, como aquelas com a presença de um apreciável elemento de novidade e a resolução de problemas científicos e tecnológicos, cuja solução não seja aparente para alguém familiarizado com o estoque de conhecimentos básicos daquela área. 


\section{Referências}

BÁLAN, J. La competencia internacional por los talentos. Primera Revista Latinoamericana de Libros, oct./nov. 2008. Disponível em: <https://www.revistaprl.com/review. php?article=64\&edition=1-6>. Acesso em: 20 out. 2008.

BALBACHEVSKY, E. A profissão academica no Brasil: evolução recente e perspectivas futuras. São Paulo: nupes/usp, [2001]. (Projeto de pesquisa).

BALBACHEVSKY, E. Brazil's high education responses to the global challenges of the 21st century. Thinking Brazil, Washington, DC, n. 23, p. 1-4, 2006.

BALBACHEVSKY, E.; MARQUES, F. “Fuga de cerebros” en Brasil: los costos públicos del errado entendimiento de una realidad acadêmica. In: Aupetit, S.D.; GérARD, E. (Ed.). Fuga de cerebros, movilidad académica, redes científicas: perspectivas latinoamericanas. México, DF: Cinvestav, 2009. p. 161-173.

BRASIL. Ministério da Educação. Instituto Nacional de Estudos e Pesquisas Educacionais (INEP). Censo da educação superior 1998 e 2008. Disponível em: <http://www. inep.gov.br/imprensa/noticias/censo/superior/arquivo09.htm>. Acesso em: $8 \mathrm{dez}$. 2009.

COMMISSION OF THE EUROPEAN COMMUNITIES (EC). Better careers and more mobility: a European partnership for researchers. Brussels: Commission of the European Communities, 2008.

COORDENAÇÃO DE APERFEIÇOAMENTO DE PESSOAL DE NÍVEL SUPERIOR (CAPES). GEOCAPES: distribuição de discentes: 1998 e 2008. Brasília, DF: CAPES, 2009. Disponível em: <http://geocapes.capes.gov.br/>. Acesso em: 8 dez. 2009.

DAVENPORT, S. Panic and panacea: brain drain and science and technology human capital policy. Research Policy, Amsterdam, n. 33, p. 617-630, 2004.

DE MEIS, L.; LONGO, P.H. The training of Brazilian biochemists in Brazil and developed countries: costs and benefits. Biochemical Education, Amsterdam, v. 18, n. 4, p. 182-188, 1990.

EUROPEAN CENTRE FOR THE DEVELOPMENT OF VOCATIONAL TRAINING (GEDEFOP). Skill needs in Europe: focus on 2020. Luxembourg: Official Publications of the European Communities, 2008.

EUROPEAN UNION RESEARCH ADVISORY BOARD (EURAB). Background document of the Working Group on Increasing the Attractiveness of Science, Engineering $\mathcal{E}$ Technology Careers, Sept. 2002. 
GLÄNZEL, W.; LETA, J.; THUS, B. Science in Brazil. part 1: a macro-level comparative study. Scientometrics, Budapest, v. 67, n. 1, p. 67-86, 2006.

GUIMARÃES, R. A diáspora: um estudo exploratório sobre o deslocamento geográfico de pesquisadores brasileiros na década de 90. Dados [online], v. 45, n. 4, p. 705-750, 2002. Disponível em: $<$ http://www.scielo.br/pdf/dados/v45n4/a06v45n4.pdf >. Acesso em: 8 dez. 2009.

HEIDRICK \& STRUGGLES INTERNATIONAL INC, ECONOMIST INTELLINGENCE UNIT. Mapping of global talent: essays and insights. [s.l.: s.n] 2007.

INSTITUTO BRASILEIRO DE GEOGRAFIA E ESTATISTICA (IBGE). Pesquisa de Inovação Tecnológica (PINTEC 2005). Rio de Janeiro: IBGE, 2007.

KATSELI, L.; LUCAS, R.; XENOGIANI, T. Effects of migration on sending countries: what do we know? Paris: OECD, 2006.

KHADRIA, B. Human resources in science and technology in India and the international mobility of highly skilled Indians. Paris: OECD, 2004. (OECD Science, Technology and Industry Working Papers, n. 7).

MAHROUM, S. Highly skilled globetrotters: mapping the international migration of human capital. RED Management, Malden, v. 30, n. 1, p. 23-32, 2000.

MENEGHINI, R. Performance of Brazilian scientists with previous Ph.D. training in Brazil and in developed countries: the case of chemists. Ciência \& Cultura, São Paulo, n. 43, p. 342-346, 1991.

MEYER, J.B. Policy implications of the brain drain's changing face. Policy Briefs, Science and Development Network, 2003.

MEYER, J.B.; BROWN, M. Scientific diasporas: a new approach to the brain drain. In: UNESCO-ICSU WORLD CONFERENCE ON SCIENCE, Budapest, Hungary, 26 June-1 July 1999.

MEYER, J.B.; KAPLAN, D.; CHARUM, J. El nomadismo científico y la nueva geopolítica del conocimiento. International Social Science Journal, Paris, n. 168, p. 309-321, 2001.

MOURA CASTRO, C. Brain drain in Latin America: myth and reality. [s.l: s.n.], 2007. Disponível em: <http://www.claudiomouracastro.com.br/upload/Brain\%20drain\%20 in\%20Latin\%20America\%20myth\%20and\%20reality.pdf> Acesso em: 22 nov. 2008.

NATIONAL ACADEMY OF SCIENCES (NAS). Careers in science and technology: an international perspective. Washington, DC: NAP, 1995. 
NATIONAL SCIENCE FOUNDATION (NSF). Survey of earned doctorates 2008. Arlington, VA: NSF, 2009a.

NATIONAL SCIENCE FOUNDATION (NSF). S\&E graduate enrollments accelerate in 2007; enrollments of foreign students reach new high. InfoBrief, NSF 09-314, 2009b.

NERAD, M. Globalization and its impact on research education: trends and emerging best practices for the doctorate of the future. In: KileY, M.; Mullins, G. (Ed.). Quality in postgraduate research: knowledge creation in testing times. Canberra: CEDAM, The Australian National University, 2006. p. 5-12.

ORGANISATION FOR ECONOMIC CO-OPERATION AND DEVELOPMENT (OECD). Human resources for science, technology and innovation in China. In: $O E C D$ Reviews of Innovation Policy: China. Paris: oecD, 2008. cap. 6, p. 305-345.

ORGANISATION FOR ECONOMIC CO-OPERATION AND DEVELOPMENT (OECD). International Migration Outlook: sopemi 2009. Paris: oecD, 2009.

SANTANA LOMBAS, M.L. Há indícios de brain drain no Brasil?: um estudo sobre doutorandos no exterior. 1999. 116p. Dissertação (Mestrado em Educação) - Universidade de Brasília, Brasília, DF.

SCHWARTZMAN, S. (Coord.). Projeto Retorno: avaliação do impacto do treinamento no exterior de pessoal qualificado. Rio de Janeiro: EBAP/FGV, 1972. (Relatório final). Disponível em: <http://www.schwartzman.org.br/simon/proj_retorno.htm>. Acesso em: 12 set. 2008 .

SCHWARTZMAN, S. Brain drain: pesquisa multinacional? In: Oliveira Nunes, E. (Org.). A aventura sociológica: objetividade, paixão, improviso e método na pesquisa social. Rio de Janeiro: Zahar, 1978. p. 67-85.

SCHWARTZMAN, S. Nacionalismo versus internacionalismo en las políticas de formación de recursos humanos de alto nível. In: Aupetit, S.D.; Gérard, E. (Ed.). Fuga de cerebros, movilidad académica, redes científicas: perspectivas latinoamericanas. México, dF: Cinvestav, 2009. p. 63-73.

SOLIMANO, A. The international mobility of talent and its impact on global development: an overview. CEPAL Serie Macroeconomía del Desarrollo, Santiago, n. 52, p. 1-35, 2006a.

SOLIMANO, A. Mobilizing talent for global development. UNU-WIDER Policy Brief, Helsinki, n. 7, p. 1-8, 2006b.

STATISTICAL OFFICE OF THE EUROPEAN UNION (EUROSTAT). Annual data on human resources in science $\mathcal{E}$ technology (HRST) and sub-groups of HRST at the national 
level. 2009. Disponível em: <http://epp.eurostat.ec.europa.eu/portal/page/portal/science_technology_innovation/data/database >. Acesso em: 16 out. 2009.

VELHO, L. Formação de doutores no país e no exterior: estratégias alternativas ou complementares? Dados [online], v. 44, n. 3, p. 607-631, 2001. Disponível em: <http:// www.scielo.br/pdf/dados/v44n3/a05v44n3.pdf>. Acesso em: 8 out. 2008.

VELLOSO, J. Pós-Graduação: egressos, trabalho e formação no país e no exterior. In: Steiner, J.E.; Malnic, G. (Org.). Ensino superior: conceito e dinâmica. São Paulo: EDUSP, 2006.

VESSURI, H. Training of researchers in Latin America and the Caribbean. In: MolLIS, M.; Nussbaum Voent, M. (Ed.). Research and higher education policies for transforming societies: perspectives from Latin America and the Caribbean; selected proceedings. Paris: UNESCO, 2007. p. 141-152.

Recebido em 28 de janeiro de 2010.

Aprovado em 10 de agosto de 2010. 